\title{
Aplicación de la Técnica de Diagnóstico por Imágenes en la Evaluación Funcional de Sistemas Electrónicos de Aceleración
}

\section{Application of the Diagnostic Imaging Technique in the Functional Evaluation of Electronic Acceleration Systems}

\author{
Cristian Lema Romero \\ Instituto Superior Tecnológico \\ Luis Rogerio González \\ Azogues, Ecuador \\ fernando.lema@institutoscanar.ec \\ Orcid: 0000-0002-1618-3214
}

\author{
Rómulo Astudillo Bravo \\ Instituto Superior Tecnológico \\ Luis Rogerio González \\ Azogues, Ecuador
}

\author{
Mayra Asitimbay Regalado \\ Instituto Superior Tecnológico \\ Luis Rogerio González \\ Azogues, Ecuador
}

romulo.astudillo@institutoscanar.ec mayra.asitimbay@institutoscanar.ec

Orcid: 0000-0001-9001-5270

\begin{abstract}
Resumen - Con el desarrollo de la técnica de diagnóstico por imágenes aplicado en el campo automotriz se puede poner a prueba actuadores, sensores, y demás elementos electrónicos del vehículo. Esta técnica se basa en la aplicación de corriente alterna de baja intensidad en el elemento que se va a comprobar, donde la circulación de esta corriente referenciada a la masa del circuito permite graficar una imagen del comportamiento en el desfase de la tensión y corriente. La captura de la señal se puede realizar en cualquier parte del componente o circuito y también en los pines de entrada y salida en cualquier componente eléctrico o electrónico automotriz. Para aplicar la técnica se desarrolla un circuito electrónico que permite utilizar un osciloscopio con la función XY. El estudio se realiza en un sistema de aceleración electrónico compuesto por pedal y cuerpo de aceleración; éstos internamente cuentan con sensores de posición de tipo potenciómetro. El cuerpo de aceleración cuenta con un motor eléctrico que mueve un sistema de engranajes, donde para lograr mover la aleta de aceleración y probar el comportamiento lineal de los sensores se desarrolla un control PWM. Se realizan comprobaciones especificas con la técnica de diagnóstico por imágenes y se compara con la metodología tradicional de utilizar un multímetro y un osciloscopio.
\end{abstract}

Palabras Clave: Diagnóstico por imágenes, cuerpo de aceleración electrónico, control PWM.
Abstract - The actuators, sensors and other electronic elements of a vehicle can be tested with the development of the diagnostic imaging technique applied in the automotive field. This technique is based on the application of low intensity alternating current in the element to be tested, where the circulation of this current related to the circuit mass, allows to draw an image of the behavior in the phase shift of the voltage and the current. The signal capture can be done on any part of the component or circuit and on the input and output pins on any automotive electrical or electronic component. To apply the diagnostic imaging technique, an electronic circuit is developed, allowing the use of an oscilloscope with the XY function. This study is carried out on an electronic acceleration system composed of a pedal and an acceleration body; these internally have potentiometer-type position sensors. The acceleration body has an electric motor that moves a gear system, where to achieve the acceleration flap movement and to test the linear behavior of the sensors, a PWM control is developed. Specific tests are made with the diagnostic imaging technique and compared with the traditional methodology of using a multimeter and an oscilloscope.

Keywords: Imaging diagnosis, electronic throttle body, PWM control.

Sumario: I Introducción, II Materiales y Métodos, III Resultados, IV Conclusiones.

Como citar: Lema, Cristian., Astudillo, Rómulo., \& Asitimbay, Mayra. (2020). Aplicación de la Técnica de Diagnóstico por Imágenes en la Evaluación Funcional de Sistemas Electrónicos de Aceleración. Revista Tecnológica - Espol, 32(2). Recuperado a partir de http://www.rte.espol.edu.ec/index.php/tecnologica/article/view/704 


\section{INTRODUCCIÓN}

La importancia de la técnica de diagnóstico por imágenes en la industria automotriz permite poner a prueba actuadores, sensores, y demás elementos electrónicos del vehículo. Se basa en la aplicación de corriente alterna de intensidad baja en el elemento que se va a comprobar; la circulación de esta corriente referenciada a la masa del circuito permite graficar una imagen del comportamiento en el desfase de la tensión y corriente según lo argumenta [1] [2] [3] [4].

Lo desarrollado en este trabajo permite utilizar la técnica de diagnóstico por imágenes mediante el desarrollo de un banco de comprobación para un cuerpo de aceleración electrónica y un pedal de acelerador de un vehículo Chevrolet Silverado. Se utiliza un osciloscopio automotriz de marca Hantek DSO1008A, se desarrolla un circuito que replica el trazador de imágenes propuesto por [2], una fuente de $12 \mathrm{~V}$ y $5 \mathrm{~V}$, un módulo de encendido para el accionamiento del motor eléctrico del cuerpo de aceleración y una computadora de escritorio con todos sus componentes, entre otros elementos de conexión.

El análisis realizado permite determinar el estado funcional de los sensores integrados en el pedal y en el cuerpo de aceleración mediante la caracterización de imágenes con el afán de establecer unas gráficas de referencias que sirvan de comparativa para un diagnóstico futuro sobre estos sistemas.

\section{MATERIALES Y MÉTODOS}

En el presente trabajo se utiliza una metodología de tipo analítica experimental mediante el desarrollo de un banco de comprobación para sistemas de aceleración electrónicos utilizando la técnica de diagnóstico por imágenes de acuerdo con lo propuesto en [1] [2] [3] [4] [5].

Se realiza un análisis funcional del sistema de aceleración electrónica de un vehículo Chevrolet Silverado en cuanto al pedal y aleta de aceleración electrónica, mediante la caracterización del comportamiento de los sensores y motor eléctrico internos utilizando la técnica de diagnóstico por imágenes.

Este método consiste en introducir al componente o circuito a diagnosticar una corriente alterna de muy bajo valor según se argumenta en [1], donde la circulación de esta corriente, referenciada a la masa del circuito, traza una imagen del comportamiento de los componentes electrónicos en análisis y se identifica como patrón del comportamiento electrónico. [6]

La imagen generada, de acuerdo con [1], está vinculada al desfasaje entre la onda de tensión y la onda de corriente que circula en el componente. La captura de la señal se puede realizar en cualquier parte del componente o circuito y también en los pines de entrada y salida, ya sea en los sensores o el motor eléctrico que mueve la aleta de aceleración.

En el sistema de aceleración electrónico se realizaron mediciones de voltaje en las señales de los 2 sensores de posición denominados como Throttle Position Sensor (TPS1 y TPS2), esto cuando el ciclo de trabajo de una señal de ancho de pulso modulado (Pulse Width Modulation - PWM) se incrementa progresivamente, permitiendo la apertura de la aleta y la consecuente variación en la señal de los sensores de posición de ésta. Para controlar el consumo de corriente del motor eléctrico y realizar un acoplamiento entre la señal del control PWM y el motor eléctrico, se utiliza un módulo de encendido denominado por [7] como módulo de ignición de alta energía - High Energy Ignition - (HEI). Estos módulos normalmente son utilizados en sistemas de encendido en la marca General Motors (GM) y su configuración es de 4 pines.

Se generan imágenes de estos componentes que se encuentran en estado funcional óptimo, ya que se realiza en un vehículo sin códigos de falla alguna en relación con este sistema, y se implementan estos elementos en un banco de comprobación. Se interpreta su estado funcional con base en la comparación de las señales de referencia tomadas.

En [1] se establece que la técnica permite tener una interpretación de los diferentes tipos de imágenes de tal forma que el técnico determina la falla interna del circuito a analizar. El sistema es aplicable al diagnóstico de módulos, componentes electrónicos del automóvil, redes, sensores, actuadores, etc.

Para este proceso hay que considerar que la generación de la imagen depende del tipo de circuito que esté bajo prueba [8]. Si el circuito es interrumpido o no existe circulación de corriente el resultado será una línea horizontal, o por lo contrario, si el componente bajo prueba presenta un cortocircuito la línea es vertical según lo que argumenta [3].

El circuito abierto se muestra en la Fig. 1, que tiene una resistencia infinita por lo tanto es una línea horizontal, puesto que la corriente es siempre cero independientemente del voltaje aplicado según lo explica [2]. Bajo estos criterios sería despreciable la escala de tensión y corriente que se muestra en la figura 1, siendo importante más bien el patrón generado en las pruebas que se realice.

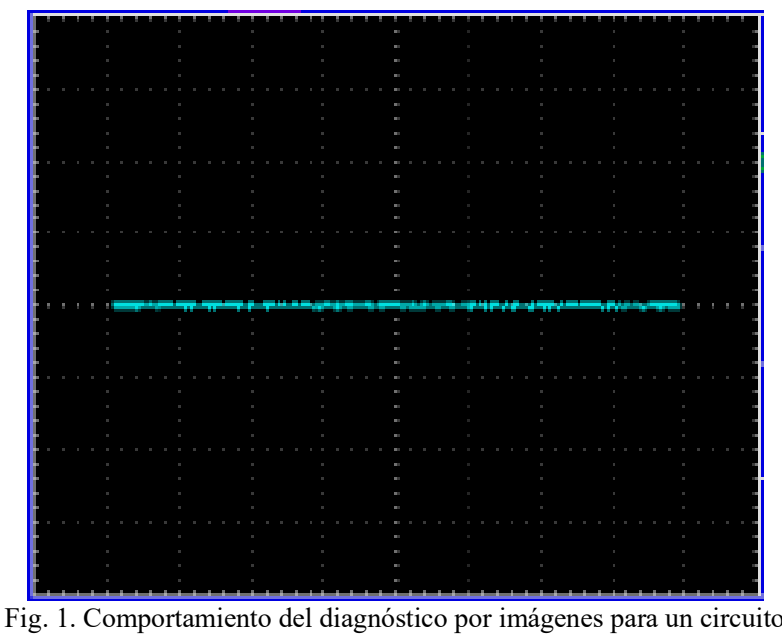

Fig. 1. Comportamiento del diagnóstico por imágenes para un circuito abierto

Un cortocircuito es una línea vertical, debido a que el flujo de corriente para cualquier tensión aplicada es infinito, se representa por una línea vertical sobre el eje de corriente debido ya que no existe voltaje en las puntas de prueba, esto se argumenta en [2]. Así mismo, la escala de tensión y corriente al igual que el criterio explicado en el 
párrafo anterior es despreciable ya que orienta a un patrón específico de comportamiento, esto se muestra en la Fig. 2.

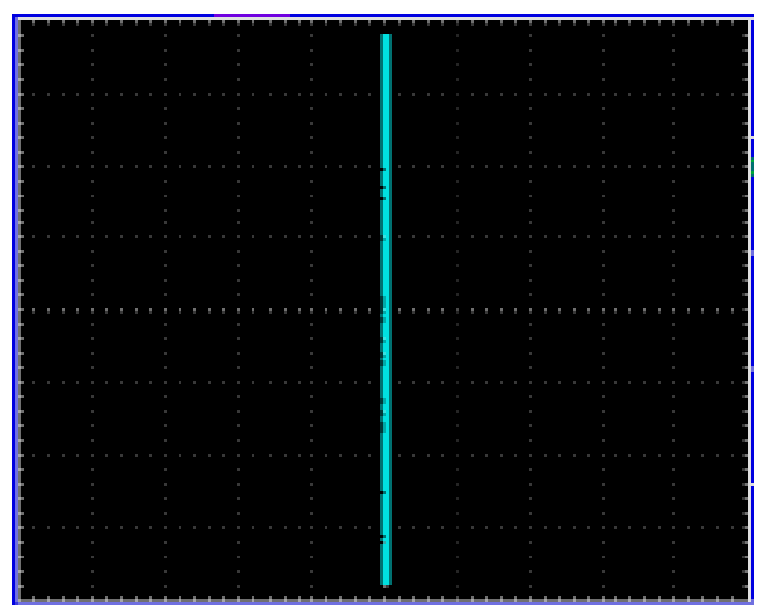

Fig. 2. Comportamiento del diagnóstico por imágenes para un corto circuito

El trazado de la curva para un elemento resistivo resulta una línea inclinada. Esta forma se produce debido a las ondas senoidales de intensidad y voltaje en la resistencia varían según el grado de circulación de corriente en dependencia del valor de la resistencia según explica [4]. Se entiende en dependencia de la resistencia que se tendrá una determinada inclinación, y si se analiza un patrón de cualquier componente no sería necesario considerar la escala de tensión y corriente, sino más bien el patrón de la figura que genera el componente bajo prueba ya que se puede comparar cuando este elemento tenga falla, determinando fácilmente su desplazamiento angular y por ende su evidente defecto. Esto se evidencia en la Fig. 3.

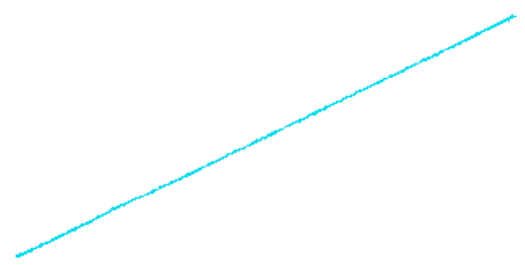

Fig. 3. Comportamiento del diagnóstico por imágenes para un elemento resistivo

El trazo de la curva para un elemento capacitivo indica una forma elíptica, esta forma se produce debido a un desfase que existe de 90 grados en la onda senoidal del voltaje con respectos a la onda senoidal de la intensidad; el ancho de la forma elíptica indicará el valor capacitivo de acuerdo con [4]. Se muestra en la Fig. 4.

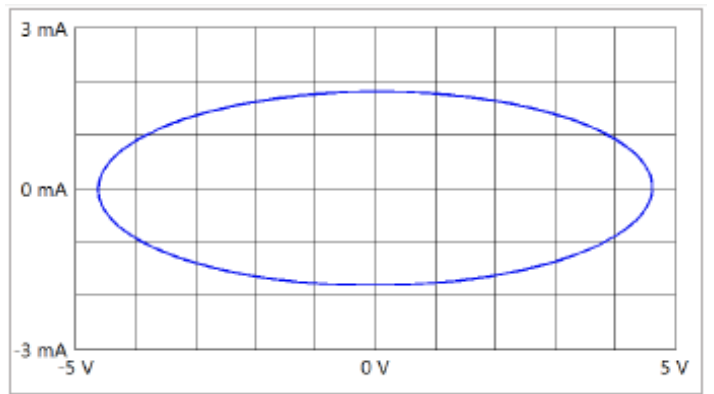

Fig. 4. Comportamiento del diagnóstico por imágenes para un elemento capacitivo. Fuente: [4]
El trazado de curva según lo explica [4] para un elemento semiconductor (diodo) indica una forma de $\mathrm{L}$ como se observa en la Fig. 5 en a) y para el caso de un diodo Zener se observa una forma de dos L invertidas en b). Se muestra en la figura 5 a)

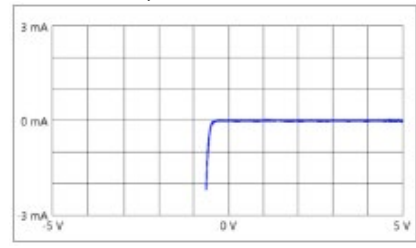

Fig. 5. Imágenes para elementos semiconductor. a) Diodo, b) Diodo Zener. Fuente: [4]
Dependiendo del fabricante, el pedal del acelerador cuenta con dos o tres sensores que miden la posición ya sea del pedal o del cuerpo de aceleración. Al presionar el pedal, se envía una señal analógica a la computadora de motor, la cual interpreta la solicitud del conductor y ordena al cuerpo de aceleración la apertura de la mariposa, en función del requerimiento y de las condiciones de desempeño del vehículo [9]. Fabricantes como General Motors (GM) y Ford utilizan hasta tres sensores para la verificación de la posición exacta del pedal. El pedal y cuerpo de aceleración en estudio consta de dos sensores de posición interna, a los cuales se les conoce con la nomenclatura Accelerator Pedal Position (APP1 y APP2); estos sensores sirven para el monitoreo de la posición exacta del pedal según [9] y (TPS1, TPS2) para los sensores de posición de aleta de aceleración.

\section{RESULTADOS}

La construcción del circuito se realiza de acuerdo con lo que se establece en la metodología. El circuito que se sugiere en [2] y[10]:B]'] es adaptado para el estudio y replica el proceso de diagnóstico por imágenes, con una adaptación que permite el uso de un osciloscopio normal de la marca Hantek 1008C según sugieren[11] [12] Para la fuente de corriente alterna se utiliza un transformador de 120Voltios de Corriente Alterna (VAC) a $12 \mathrm{VAC}$, el cual se muestra en la Fig. 6.

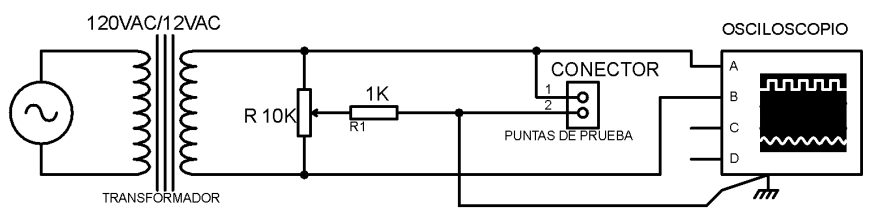

Fig. 6. Diagrama de trazador de imágenes Fuente: Adaptado de [2]

El procedimiento de construcción requiere la adquisición de los componentes y materiales para su fabricación, tales como estaño, cautín, pasta de soldar, placa perforada, conos banana, el transformador de corriente, resistencia de $1 \mathrm{Kohm}$ y potenciómetro de $10 \mathrm{KOhm}$. Se ubican los componentes en una caja donde se podrá manipular y realizar las pruebas con la técnica desarrollada, lo cual se muestra en la Fig. 7. 


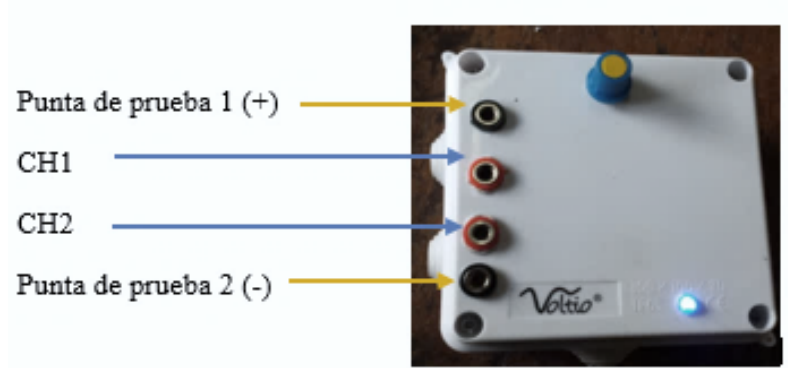

Fig. 7. Trazador de imágenes desarrollado

La configuración del pedal se muestra en la Fig. 8 en la forma de la disposición de sus pines. Estos pines de izquierda a derecha en vista frontal del conector llevan $+5 \mathrm{~V}$ de alimentación para los dos sensores de posición, una señal del primer sensor de posición de la aleta y a continuación una masa. El cuarto pin dispone una masa para el segundo sensor, y a continuación la señal del segundo sensor de posición de la aleta de aceleración. Finalmente, en el pin seis existe la alimentación de $+5 \mathrm{~V}$ para el segundo sensor de posición.

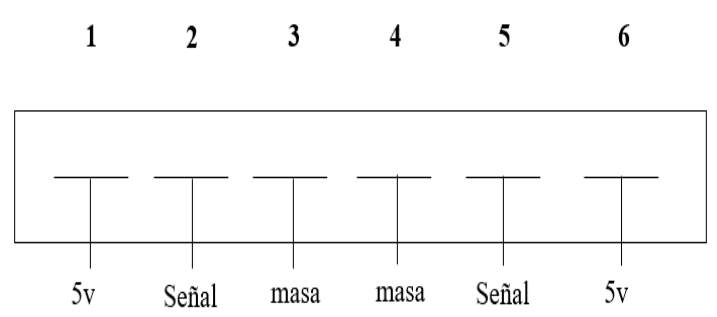

Fig. 8. Disposición de pines del pedal electrónico

La configuración del cuerpo de aceleración electrónico se muestra en la Fig. 9 en forma de la disposición de pines; éstos, de izquierda a derecha en vista frontal del conector llevan una alimentación de $+5 \mathrm{~V}$ del TPS2, una señal del primer sensor de posición de la aleta y a continuación otra señal del segundo sensor de posición; el cuarto pin corresponde a una masa del TPS1. En la fila inferior, en el primer pin, se identifica una masa del TPS2, y seguidamente el pin de control del motor eléctrico del cuerpo de aceleración, el pin de alimentación de $+5 \mathrm{~V}$ del TPS1 y el último nuevamente el pin de control del motor.

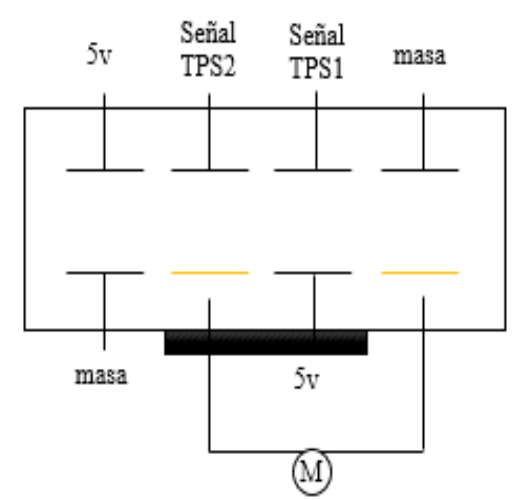

Fig. 9. Disposición de pines del cuerpo de aceleración

Se mide las señales de los sensores utilizando una técnica convencional de diagnóstico mediante el osciloscopio en las diferentes aperturas aproximadas al $25 \%, 50 \%, 75 \%, 100 \%$ de control PWM aplicado para las señales de TPS y de movimiento del pedal para las señales APP. Se identifica la variación de voltaje en dicha señal. Como ejemplo se muestra la señal del pedal APP1 ascendente al $25 \%$ de apertura que inicia con un valor de 0,84 voltios, lo cual se muestra en la Fig. 10. La escala de la figura capturada por el osciloscopio muestra en el eje X el tiempo en una escala de 500ms por división y en el eje $\mathrm{Y}$ una escala de Tensión $(\mathrm{V})$.

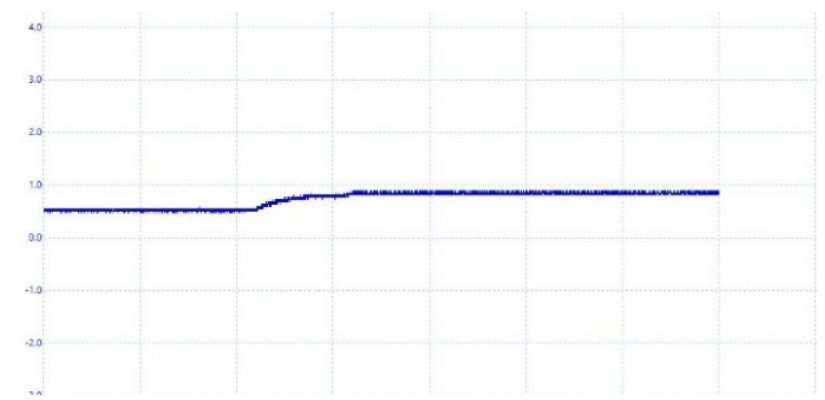

Fig. 10 Accionamiento del pedal al 25\%

Se nota que el potenciómetro se encuentra completamente funcional debido a que no presenta ninguna deformación ni entrecorte a lo largo del desplazamiento del pedal, desde la posición de reposo hasta el desplazamiento total. Sucede lo mismo con las señales de los otros sensores de posición analizados tanto en el pedal como en el cuerpo de aceleración.

Los resultados, aplicando el método de diagnóstico por imágenes obtenidos al pedal de aceleración señal del APP1 entre los pines dos (señal) y tres (masa), se muestran en la Fig. 11. La pendiente de la recta que se obtiene dependerá directamente del valor de resistencia eléctrica del sensor, por lo que con la apertura del $25 \%$ aplicado se obtiene siempre este patrón de imagen, teniendo un valor específico para esta prueba. Siendo así, no es necesario tener la escala en los ejes XY sino más bien el patrón para que de esta forma, cuando se compare con otro componente de las mismas características, se pueda valorar el elemento solo con el patrón de la imagen obtenida más no con su comportamiento de tensión y corriente. De esta forma, la técnica de diagnóstico por imágenes permite tener una base de datos de múltiples componentes en condiciones de operación específicas para que se pueda comparar elementos con sospecha de fallo.

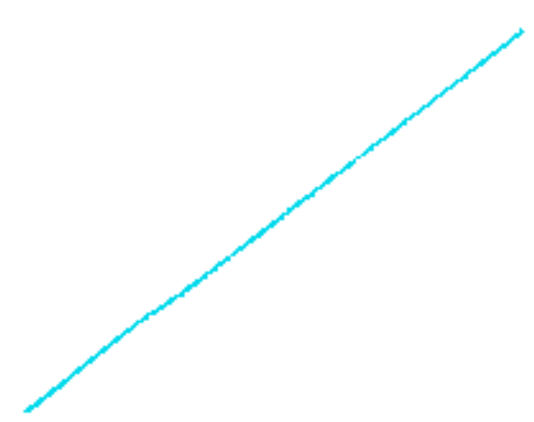

Fig. 11. Señal APP1 pines 2 y 3 utilizando la técnica de diagnóstico por imágenes

Los resultados aplicando la técnica de diagnóstico por imágenes al tomar las muestras en el sensor de posición 
TPS1 con un $25 \%$ de PWM se identifican en la Fig. 12. La figura obtenida corresponde con una resistencia y ésta varía en función de la apertura de la aleta. Se obtiene el mismo comportamiento en ambos sensores.

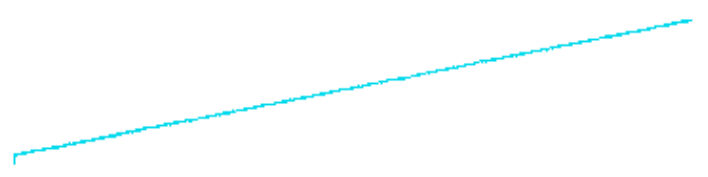

Fig. 12. Figura por medio de diagnóstico por imágenes en el sensor TPS 1

El proceso de diagnóstico por imagen empleando el trazador de curvas tiene limitaciones en la visualización de imágenes en circuitos donde la resistencia es muy baja o alta. Las pruebas efectuadas en el motor eléctrico del cuerpo de aceleración muestran un valor muy cercano a un cortocircuito, ya que su resistencia se encuentra en valores cercanos a 3ohmios. Se recomienda caracterizar su comportamiento con el ajuste que se puede realizar por medio del potenciómetro del trazador implementado. Éste permite un ajuste para una visualización más adecuada; sin embargo, se debe contrastar con métodos tradicionales de diagnóstico como un multímetro donde se puede determinar de forma exacta su resistencia o la medición de corriente del motor por medio de una pinza amperimétrica. En el cuerpo de aceleración en estudio se determina un valor de 3 ohmios de resistencia eléctrica.

\section{CONCLUSIONES}

El control PWM desarrollado se mantiene a una frecuencia fija de $1 \mathrm{Khz}$ y se varía el ancho de pulso para llegar a obtener el rango de apertura de la aleta. Se determina que se encuentra entre el $55 \%$ al $75 \%$ para una apertura total de aleta bajo estas condiciones de operación en las pruebas realizadas. Con esto, la aleta de aceleración electrónica puede ser operada para su total apertura permitiendo aplicar la técnica de diagnóstico por imágenes y caracterizando su comportamiento.

Se registra la figura típica de una resistencia eléctrica en los sensores TPS y APP utilizando la técnica de diagnóstico por imágenes; esto según lo descrito en la metodología donde la variación de dicha resistencia se encuentra en función de la posición de la aleta o pedal; sin embargo, es de notar que aplicando esta metodología no es necesario establecer los valores típicos punto a punto para determinar un cambio de señal, como se realiza con la técnica convencional mediante el osciloscopio; más bien, al variar la posición de cualquier sensor, éste simplemente registra un cambio en la pendiente de dicha gráfica y es más sencillo identificar si existe algún problema en la pista resistiva de los elementos en análisis, siendo subjetiva la escala de tensión - corriente de la técnica utilizada. Sumado a la ventaja de no necesitar alimentación para su comprobación y por consecuente no necesitar el vehículo para su respectivo análisis por técnicas convencionales de diagnosis, simplifica efectivamente el proceso para definir el recambio del componente con el uso de esta metodología, ya que se puede establecer un banco de patrones de distintos componentes.

\section{RECONOCIMIENTOS}

Este trabajo ha sido financiado parcialmente por el Proyecto de Investigación denominado "Desarrollo de un laboratorio didáctico para la evaluación funcional de sistemas de aceleración electrónicos automotrices en el Instituto Superior Tecnológico Luis Rogerio González, utilizando la técnica de diagnóstico por imágenes".

\section{REFERENCIAS}

[1] F. Augeri, "Cise Electronic," Feb. 08, 2015. [Online]. Available: http://www.cise.com/portal/notas-tecnicas/item/757diagn $\% \mathrm{C} 3 \% \mathrm{~B} 3$ stico-por$\underline{\mathrm{im} \% \mathrm{C} 3 \% \mathrm{~A} 1 \text { genes.html? tmpl=component\&print }=1}$

[2] G. Erazo, L. Murillo and E. Guangaje, "Diagnóstico por Imagen del Sistema de Control Electrónico de Inyección Diésel Riel Común CRDI," Quito: Universidad de las Fuerzas Armadas ESPE, 2016.

[3] J. P. Peñafiel, "Diseño de manual para diagnóstico por imágenes de circuitos electrónicos automotrices a través del software Fados," Guayaquil: Universidad Internacional del Ecuador, 2017.

[4] E. cajas and A. Cevallos, "Diseño e implementación de un software para la detección de fallos en los sistemas electrónicos del vehículo, aplicando la técnica de diagnóstico por imagen.," Cuenca: Universidad Politécnica Salesiana., 2017.

[5] R. Bosch, "Sistema EGAS," Bosch, Jul. 17, 2018. [Online]. Available: http://br.boschautomotive.com/es/internet/parts/parts_and_accessories_2/motor _and sytems/benzin/more sensors/sistema_egas_pedal_acelerad or_eletronico/sistema egas pedal acelerador_eletronico.html

[6] J. L. Orozco, "Sistema de Encendido Electrónico," México: Electrónica y Servicio., 2014.

[7] F. Figueroa, "Circuitos para armar," Jul. 25, 2017. [Online]. Available: https://sensoricx.com/circuitos-para-armar/reto-deldia-construye-este-trazador-de-curvas/

[8] R. A. Arrieta, "Autosoporte," Nov. 15, 2016. [Online]. Available: https://www.autosoporte.com/index.php/blogautomotriz/item/471-conoce-el-sistema-de-aceleracionelectronico

[9] M. Laloa, "Implementación de un banco de pruebas para la unidad de control electrónico de vehículos con sistemas de inyección electrónica para la Escuela de Ingeniería Automotriz," Riobamba: Escuela Superior Politécnica De Chimborazo, 2012.

[10] R. Núñez, "Diagnóstico Por Imágenes Del Sistema De Inyección Electrónico," Guayaquil: Universidad Internacional Del Ecuador, 2018. 